\title{
Oncology and Chronic Disease
}

\author{
Tilman Rohrera, Thomas M.K. Völkl ${ }^{b}$ and Helmuth G. Dörrb \\ aPediatric Endocrinology, Department of Pediatrics and Neonatology, University Medical Centre, \\ Homburg/Saar, Germany \\ bPediatric Endocrinology, Department of Pediatrics, University of Erlangen, Erlangen, Germany
}

\begin{abstract}
We are pleased to present the chapter on Oncology and Chronic Disease. Most published papers with importance to pediatric endocrinology were about late effects of cancer. This is not surprising since significant improvements in diagnosis and intensive treatment modalities of childhood cancer during the last 20 years have led to increasing survival rates worldwide. In developed countries, 1/250 of the adult population is a long-term survivor of childhood cancer. However, children who survive childhood cancer are at risk of various late effects as young adults, such as second malignant neoplasms, cardiovascular disease, endocrine disorders, and effects on fertility and reproduction. The latter are of particular interest for the surviving patients. Regarding fertility, we selected three guidelines recommending long-term follow-up of survivors. Our impression is that the number of papers published on the topic 'chronic disease' in the year 2013 was less than in 2012. As before, we analyzed this topic from two sides: (1) from the perspective of affected patients, e.g. analyzing growth and pubertal development, and (2) from the perspective of side effects on the endocrine system in relation to the respective therapeutic regimens.
\end{abstract}

\section{Late effects of childhood cancer therapy}

\section{Endocrine late sequelae in long-term survivors of childhood non-Hodgkin lymphoma}

\author{
Van Waas M, Neggers SJ, Te Winkel ML, Beishuizen A, Pieters R, van den Heuvel-Eibrink MM \\ Department of Pediatric Oncology/Hematology, Erasmus Medical Center, Rotterdam, The Netherlands \\ Ann Oncol 2012;23:1626-1632
}

Background: The aim of this study was to investigate the long-term endocrine effects of treatment of childhood non-Hodgkin lymphoma (NHL).

Methods: A single-center cohort of 84 survivors (22 females) was included in this retrospective study. Median age was 21 (9-40) years and time after cessation of therapy was 12 (4-30) years. Height, weight, percentage fat, lean body mass (LBM), bone mineral content (BMC), bone mineral density of total body $(\mathrm{BMD}(\mathrm{TB}))$ and bone mineral density of lumbar spine $(\mathrm{BMD}(\mathrm{LS}))$ were measured. Thyroidstimulating hormone (TSH), free thyroxin $\left(\mathrm{fT}_{4}\right)$, insulin-like growth factor-1 (IGF-1), inhibin B and anti-mullerian hormone (AMH) levels were measured. Results were compared with Dutch controls.

Results: Height was lower in survivors (mean standard deviation score (SDS) $-0.36, \mathrm{p}=0.002$ ), but the shorter stature was already present at diagnosis (mean SDS $-0.28, \mathrm{p}=0.023$ ). Body mass index, percentage fat, BMC, BMD(TB) and BMD(LS) were not different from controls. LBM was lower in survivors (mean SDS $-0.47, \mathrm{p}=0.008$ ). TSH, $\mathrm{fT}_{4}$ and IGF-1 were normal in all survivors. Three of 20 adult females had low AMH levels and 23 of 42 adult males had low inhibin B levels.

Conclusions: Twelve years after cessation of treatment, NHL survivors did not develop adiposity, osteoporosis or thyroid disease. Male survivors may be at risk for infertility.

The main strength of this study is that the authors report 12-year follow-up data on bone density and body composition by dual-energy X-ray absorptiometry (DXA), correcting the data for bone length and height, respectively. The second noteworthy aspect is that the study also investigated the effects of cumulative chemotherapies on inhibin B as a marker of fertility. Correction for age at diagnosis and total cumulative alkylating agents revealed that the antimetabolic cancer drug cytarabine (synonyms: cytosine arabinoside, Ara-C), which interferes with DNA synthesis rather than causing its alkylation, had a negative effect on spermatogenesis as reflected by decreased serum inhibin $B$ levels 
in male survivors of childhood NHL. The authors are the first to investigate this aspect. Like the study by Mulcahy Levy et al. discussed below, this study shows the urgent need for prospective multicenter studies to obtain robust data.

\title{
Differential effects of radiotherapy on growth and endocrine function among acute leukemia survivors: a childhood cancer survivor study report
}

\author{
Chow EJ, Liu W, Srivastava K, Leisenring WM, Hayashi RJ, Sklar CA, Stovall M, Robison LL, Baker KS \\ Department of Pediatrics, Seattle Children's Hospital and University of Washington, Seattle, WA, USA \\ ericchow@u.washington.edu \\ Pediatr Blood Cancer 2013;60:110-115
}

Background: The differential effects of cranial radiotherapy (CRT), spinal radiotherapy (SRT), and total body irradiation (TBI) on growth and endocrine outcomes have rarely been examined in combination among childhood acute leukemia survivors.

Methods: Acute lymphoblastic and myeloid leukemia survivors $(\mathrm{n}=3,467)$ participating in the Childhood Cancer Survivor Study (an ongoing cohort study of 5 -year survivors of pediatric cancers diagnosed from 1970 to 1986). Determination of self-reported height/weight, hypothyroidism, and pregnancy/live births. Results: Compared with no radiotherapy, risk estimates were consistent across outcomes (adult short stature, hypothyroidism, absence of pregnancy/live birth) with CRT treatment associated with 2- to 3-fold increased risks, TBI associated with 5- to 10-fold increased risks, and CRT+TBI associated with $>10$-fold increased risks. Exposure to any SRT further increased risk of these outcomes 2- to 3-fold. Changes in body composition were more nuanced as CRT only was associated with an increased risk of being overweight/obese (OR 1.6, 95\% CI 1.3-1.9), whereas TBI only was associated with an increased risk of being underweight (OR 6.0, 95\% CI 2.4-14.9).

Conclusions: Although patients treated with CRT+TBI were at greatest risk for short stature, hypothyroidism, and a reduced likelihood of pregnancy/live birth, those treated with either modality alone also had significantly increased risks, including altered body composition. Any SRT exposure further increased risk in an independent fashion.

\begin{abstract}
In the past, radiotherapy was an essential cornerstone of treatment in childhood leukemia. TBI is part of allogeneic hematopoietic stem cell transplantation (HSCT). It is known that both treatments are associated with impairment of fertility in a dose-response fashion. This study investigated specific endocrine complications and their associations with particular aspects of radiotherapy, separately and in combination, against the background of the exposed body regions following CRT, SRT, or TBI. The limitations of the study are: self (or proxy) reporting of outcome data, incomplete information on HSCT conditioning regimens, and lack of hormonal data (no differentiation between primary and central hypothyroidism). Nevertheless, even if cranial and craniospinal irradiation are nowadays no longer routinely administered for acute leukemia but limited to high-risk protocols, the long-term data reported by Chow and colleagues provide a useful basis for counseling patients on specific longterm endocrine sequelae of various forms of radiotherapy.
\end{abstract}

\section{Late effects of total body irradiation and hematopoietic stem cell transplant in children under 3 years of age}

Mulcahy Levy JM, Tello T, Giller R, Wilkening G, Quinones R, Keating AK, Liu AK

Center for Cancer and Blood Disorders, Children's Hospital Colorado, Aurora, CO, USA

jean.mulcahy-levy@childrenscolorado.org

Pediatr Blood Cancer 2013;60:700-704

Background: Total body irradiation (TBI) is an important component of hematopoietic stem cell transplant (SCT) for pediatric malignancies. With increasing survival rates, late effects of SCT become more important. Younger children may be at particular risk of late effects of radiation and SCT.

Methods: The authors retrospectively reviewed outcomes of children $<3$ years of age who received TBI as part of their preparative regimen for SCT at Children's Hospital Colorado. Clinical information including the date of last follow-up, most recent laboratory values, and physiologic tests were extracted from the medical record. 
Results: Of 81 patients, who underwent SCT, 19 received TBI and of those, 15 were long-term survivors available for review. Late effects occurring in greater than $50 \%$ of the children included abnormalities involving endocrine, metabolic, renal, cataracts, and neurocognitive systems. Other organs involved less commonly included liver, skeletal, and cardiac abnormalities. Solid tumors were a rare finding with only 1 patient developing a benign osteochondroma and no identified secondary malignancies.

Conclusions: TBI has been shown to be an important part of the preparative regimen for patients undergoing SCT. The results, similar to other studies, suggest that TBI in patients $<3$ years of age will likely result in multi-organ dysfunction including endocrine, metabolic, renal, eye, and neurocognitive abnormalities. A longitudinal study with standardized testing of these systems would further clarify the late effects concerns in this patient population.

Mulcahy Levy and colleagues present data on a very young patient population receiving TBI followed by SCT for pediatric malignancies. Their findings include an increased likelihood of multi-organ dysfunction, affecting the endocrine system, metabolism, kidneys, eyes, and neurocognitive functions. However, there are limitations to this study, which lie in the retrospective nature of data collection, the small number of cases, the single-center design, and the absence of a control group of children with leukemia who did not undergo TBI.

\section{Confirmation of previous results}

\section{Langerhans' cell histiocytosis: $\mathbf{4 0}$ years' experience}

Maria Postini A, del Prever AB, Pagano M, Rivetti E, Berger M, Asaftei SD, Barat V, Andreacchio A, Fagioli F

Stem Cell Transplantation and Cellular Therapy Division, University of Turin, Turin, Italy

annapostini@hotmail.it

J Pediatr Hematol Oncol 2012;34:353-358

Background: This study analyzes 40 years' experience of Langerhans' cell histiocytosis in pediatric patients.

Methods: Between June 1968 and December 2009, 121 patients (79 males, 42 females; median age 4.13 years) were diagnosed and treated at one center (74\% monosystemic disease; $26 \%$ multisystemic). The authors evaluated the response, the survival, and the neuroendocrinological sequelae.

Results: Overall survival (OS) for all patients was $93 \%$ at 10 years from diagnosis; event-free survival (EFS) $77 \%$. OS for patients $<2$ years and $\geq 2$ years was 82 and $97 \%(p=0.003)$; EFS 48 and $87 \%(p=$ 0.001). OS for patients diagnosed before and after April 1, 1991 was 84 and 98\% ( $\mathrm{p}=0.007$ ), EFS 66 and $85 \%(\mathrm{p}=0.03)$. OS for monosystemic and multisystemic disease was 100 and $71 \%(\mathrm{p}<0.001)$; EFS 88 and $45 \%(\mathrm{p}<0.001)$. OS for 'risk' patients (involvement of bone marrow, spleen, liver, lungs) and 'low-risk' patients was 50 and 94\% ( $\mathrm{p}=0.007)$, EFS 37 and $54 \%(\mathrm{p}=0.06) .14$ patients developed diabetes insipidus, 7 growth hormone deficiency, 2 hypothyroidism, and 1 neurodegeneration.

Conclusions: This study confirms the improvements in pathogenetic knowledge and treatment over the last 20 years. Age at diagnosis $\geq 2$ years and standardized treatment are associated with improved prognoses. Multisystem involvement, especially with 'risk' organs, seems to be correlated with a worse outcome.

Whether Langerhans' cell histiocytosis (LCH) represents a reactive or a neoplastic disease is still a matter of debate. LCH is probably the only pathologic combination between oncogenesis and chronic immune dysregulation. Endocrine abnormalities are well-recognized sequelae of childhood LCH. The damage to the neuroendocrine system often manifests as central diabetes insipidus. Anterior pituitary deficits, primarily growth hormone deficiency, are also common complications of LCH [1-3]. Depending on which organs are implicated, LCH may prove rapidly fatal, develop a chronic pattern or resolve spontaneously. Diverse therapeutic approaches may be considered depending on the affected organ, including surgery, radiotherapy, and chemotherapy. The authors confirm previous studies and demonstrate an increase in the overall survival rates of all patients within the last 20 years, particularly in children $\geq 2$ years. 


\section{Long-term outcome of hypothalamic pituitary tumors in Langerhans' cell histiocytosis}

Fahrner B, Prosch H, Minkov M, Krischmann M, Gadner H, Prayer D, Grois N

St. Anna Children's Cancer Research Institute, Vienna, Austria

bernhard.fahrner@ccri.at

Pediatr Blood Cancer 2012;58:606-610

Background: Hypothalamic-pituitary (HP) disease is the most common CNS manifestation of Langerhans' cell histiocytosis (LCH) frequently leading to diabetes insipidus (DI) and anterior pituitary hormone deficiencies (APD). On MRI, loss of the normal posterior pituitary signal and thickening of the pituitary stalk have been described, as well as neurodegenerative signal changes associated with neuropsychological disabilities in some patients. The influence of therapy on the long-term course of HP tumors and neurodegeneration (ND) is not well understood.

Methods: In this retrospective survey the authors focused on patients with LCH and HP disease with clinical and MRI data available at diagnosis of HP disease and at least three follow-up investigations. Clinical and MRI follow-up information for central review and analysis were collected.

Results: 22 patients with HP tumors (HPT) registered at the LCH study center were identified. Many different treatment regimens were applied for variable periods, with more than one regimen in most patients. Regression of the tumor was seen in the majority, but all patients had APD or ND on MRI at last follow-up. In none of the patients did APD and ND regress or resolve. A deterioration of radiological ND was noted in 17 patients leading to overt clinical neuropsychological impairment in 5 patients. Conclusions: Patients with HPT appear to be at high risk to develop permanent neuroendocrine consequences. Coordinated studies for patients with LCH and HP disease including thorough MRI monitoring and neuropsychological tests are needed.

Thickening of the pituitary stalk and absence of posterior pituitary hyperintensity on MRI are markers of local LCH-related damage. The authors conclude that patients with evidence of tumorous HP disease should be followed in the context of clinical studies. A standardized diagnostic program should include regular MRI investigations over many years to monitor the evolution of tumorous lesions and radiological neurodegeneration.

\section{Important for clinical practice}

\section{Adolescent and young adult oncology: clinical practice guidelines in oncology}

Coccia PF, Altman J, Bhatia S, Borinstein SC, Flynn J, George S, Goldsby R, Hayashi R, Huang MS, Johnson RH, Beaupin LK, Link MP, Oeffinger KC, Orr KM, Pappo AS, Reed D, Spraker HL, Thomas DA, von Mehren M, Wechsler DS, Whelan KF, Zebrack BJ, Sundar H, Shead DA UNMC Eppley Cancer Center at The Nebraska Medical Center, Omaha, NE, USA J Natl Compr Canc Netw 2012;10:1112-1150

Background: Cancer is the leading cause of death among the adolescent and young adult (AYA) population, excluding homicide, suicide, or unintentional injury.

Methods and Results: The National Comprehensive Cancer Network Guidelines include psychosocial assessment, a discussion of infertility risks associated with treatment and options for fertility preservation, genetic and familial risk assessment for all patients after diagnosis, screening and monitoring of late effects in AYA cancer survivors after successful completion of therapy, and palliative care and end-oflife considerations for patients for whom curative therapy fails.

Conclusions: AYA patients should be managed by a multidisciplinary team of healthcare professionals who are well versed in the specific developmental issues relevant to this patient population.

This paper summarizes various consensus statements regarding the approaches to cancer treatment in adolescent and young adult patients (defined as age range 15-39). This group of patients has not 
shown any significant improvement in outcomes during the last years. The consensus emphasizes the importance of fertility preservation as an essential part of the management, whereupon the risk of infertility due to cancer therapy should be discussed at the time of diagnosis. The authors refer to the online Fertile Hope Risk Calculator (http://www.fertilehope.org), which is provided by LIVESTRONG, a registered trademark of the Lance Armstrong Foundation. The screening recommendations for AYA cancer survivors were adapted from the treatment exposure-based Children's Oncology Group (COG) Long-Term Follow-Up Guidelines for Survivors of Childhood, Adolescent, and Young Adult Cancers [4]. Those guidelines were also released on the internet (www.survivorshipguidelines.org).

\title{
Semen analysis in adolescent cancer patients prior to bone marrow transplantation: when is it too late for fertility preservation?
}

\author{
Nahata L, Cohen LE, Lehmann LE, Yu RN \\ Division of Endocrinology, Department of Medicine, Children's Hospital Boston, Boston, MA, USA \\ leena.nahata@childrens.harvard.edu \\ Pediatr Blood Cancer 2013;60:129-132
}

Background: Sperm banking is an effective method of fertility preservation in adolescent boys with cancer but is strikingly underutilized, partly due to inconsistencies in fertility counseling and unclear guidelines regarding who should bank sperm. Patients undergoing bone marrow transplantation (BMT) are of particular interest given the high risk of infertility in this population.

Methods: The authors reviewed the charts of male cancer patients who underwent BMT at age $\geq 13$ years at the Dana-Farber Cancer Institute (DFCI) from 2003 to 2010 to determine the number of fertility preservation attempts prior to initial treatment and/or BMT, and the outcomes of those sperm-banking attempts.

Results: 68 male cancer patients who had a BMT at age $\geq 13$ years at the DFCI from 2003 to 2010 were included in the analysis. 6 patients had attempted sperm banking prior to initial therapy. 33 patients attempted to bank prior to BMT; of those, 39\% were azoospermic and $15 \%$ were oligospermic. 19 patients did not attempt to bank, and in 13 patients the decision to bank was unclear.

Conclusions: A more consistent approach to fertility counseling is essential for adolescent cancer patients. Though first-line therapy may be low-risk in terms of long-term impact on fertility, these results demonstrate that transient gonadal dysfunction is common and ongoing chemotherapy may affect spermatogenesis. Should a patient undergo BMT during this period, sperm banking is unlikely to be successful; initial fertility risk assessment should account for this possibility.

Since spermatogenesis is often impaired after cancer therapy, sperm banking has become an effective method of fertility preservation in adolescent boys. The data presented in this paper clearly show that adequate fertility counseling is extremely important. The authors suggest that all patients should receive fertility counseling at the time of diagnosis, and to develop a multidisciplinary approach which may ultimately increase the number of patients who choose to bank sperm.

\section{Follow-up on Yearbook 2012 papers \\ The role of AMH as a marker of gonadotoxicity}

\section{Pretreatment anti-mullerian hormone levels determine rate of post-therapy ovarian reserve recovery: acute changes in ovarian reserve during and after chemotherapy}

Dillon KE, Sammel MD, Prewitt M, Ginsberg JP, Walker D, Mersereau JE, Gosiengfiao Y, Gracia CR

Department of Obstetrics and Gynecology, University of Pennsylvania School of Medicine, Philadelphia, PA, USA Fertil Steril 2013;99:477-483

Background: To identify factors associated with ovarian reserve impairment during and immediately after chemotherapy. 
Methods: Prospective cohort study; four university hospitals. Measurements of serum follicle-stimulating hormone, luteinizing hormone, estradiol, inhibin $\mathrm{B}$, and anti-mullerian hormone (AMH) as well as antral follicle counts and mean ovarian volume at 3-month intervals.

Patients: 46 adolescent and young adult women with a new diagnosis of cancer requiring chemotherapy. Changes in ovarian reserve were quantified for both the acute impact of treatment using linear regression and the longitudinal recovery after therapy using mixed-effects models adjusted for baseline ovarian reserve, use of alkylating agent, and hormone use. The women had at least one pretreatment and two posttreatment study visits (mean follow-up interval: 12 months).

Results: All measures of ovarian reserve demonstrated statistically significant changes during chemotherapy. Alkylating agent exposure and baseline ovarian reserve were acutely associated with the magnitude of impairment, and pretreatment $\mathrm{AMH}$ levels were associated with the rate of recovery of AMH after treatment. In adjusted models, participants with a pretreatment AMH level $>2 \mathrm{ng} / \mathrm{ml}$ recovered at a rate of $11.9 \%$ per month after chemotherapy, whereas participants with pretreatment AMH levels $\leq 2$ $\mathrm{ng} / \mathrm{ml}$ recovered at a rate of $2.6 \%$ per month after therapy.

Conclusions: Baseline ovarian reserve and alkylating agent exposure affect the magnitude of acute changes in ovarian reserve from chemotherapy. The rate of recovery of AMH is impacted by pretreatment levels. This should be considered during pretreatment fertility preservation counseling.

\title{
Acute onset of ovarian dysfunction in young females after start of cancer treatment
}

\author{
Morse $\mathrm{H}$, Elfving $\mathrm{M}$, Lindgren A, Wolner-Hanssen $\mathrm{P}$, Andersen CY, Ora I \\ Department of Pediatric Oncology and Hematology, Clinical Sciences, Lund University, Lund, Sweden \\ Pediatr Blood Cancer 2013;60:676-681
}

Background: Female childhood cancer survivors are at risk of ovarian failure and premature ovarian insufficiency. The authors present an interim analysis of a prospective observational study of ovarian function during cancer treatment of young females in relation to clinical factors.

Methods: 34 consecutive female cancer patients aged 0-18 years were included after informed consent. Serum or plasma levels of anti-mullerian hormone $(\mathrm{AMH})$, inhibin B, FSH, LH, and estradiol $\left(\mathrm{E}_{2}\right)$ were measured at diagnosis and every 3-4 months during and after treatment.

Results: All patients had detectable AMH levels at diagnosis. 11 patients had reached menarche (mean age 14.5 years) and the remaining patients had a mean age of 6.5 years. They all showed a rapid decline in AMH after 3 months of treatment, regardless of AMH at diagnosis, age, menarche status, or treatment. Those given radiotherapy below the diaphragm and/or stem cell transplantation (SCT) $(\mathrm{n}=9)$ had no ovarian recovery during or 1.5 years after treatment. However, recovery was observed in those given standard treatment for acute lymphoblastic leukemia $(\mathrm{n}=7)$ already during maintenance chemotherapy. For the remaining patients, longer follow-up is required for analysis of ovarian recovery after treatment.

Conclusions: Rapid ovarian dysfunction is observed in all females after initiation of cancer treatment as measured by AMH and inhibin B. The data regarding those who require abdominal radiotherapy and/ or SCT confirms the recommendations in the Nordic countries where these patients are eligible for cryopreservation of ovarian cortical tissue before start of cancer treatment.

Anti-mullerian hormone (AMH) is produced solely in the granulosa cells of growing ovarian follicles. The AMH serum levels correlate strongly with the number of growing follicles. In the Yearbook 2012, we cited two papers by Brougham et al. [5] who found that AMH levels recovered only in the low/ medium risk groups after completion of treatment, and not in the high-risk group, and by Gracia et al. [6] who found that ovarian reserve was significantly impaired in cancer survivors, even in those with regular menstrual cycles or those with normal FSH levels [6]. The two papers in 2013 by Dillon et al. and Morse et al. confirm and extend previous results in the literature showing that AMH can be used as a fertility marker to study ovarian oocyte reserve and thereby gonadal function. 


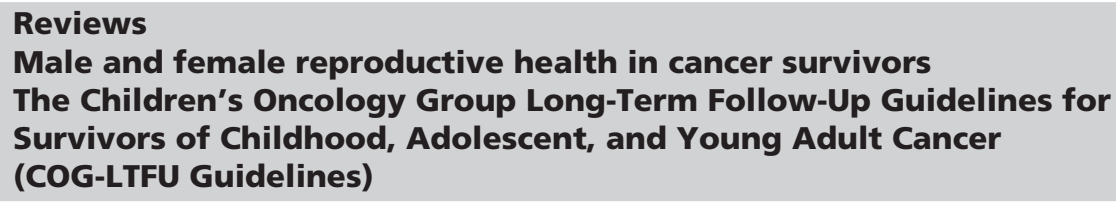

\section{Male reproductive health after childhood, adolescent, and young adult cancers: a report from the Children's Oncology Group}

Kenney LB, Cohen LE, Shnorhavorian M, Metzger ML, Lockart B, Hijiya N, Duffey-Lind E, Constine L, Green D, Meacham L

Dana-Farber Cancer Institute and Children's Hospital Boston, Boston, MA, USA

lisa_kenney@DFCI.harvard.edu

J Clin Oncol 2012:30:3408-3416

Background: The majority of children, adolescents, and young adults diagnosed with cancer will become long-term survivors. Although cancer therapy is associated with many adverse effects, one of the primary concerns of young male cancer survivors is reproductive health. Future fertility is often the focus of concern; however, it must be recognized that all aspects of male health, including pubertal development, testosterone production, and sexual function, can be impaired by cancer therapy. Although pretreatment strategies to preserve reproductive health have been beneficial to some male patients, many survivors remain at risk for long-term reproductive complications. Understanding risk factors and monitoring the reproductive health of young male survivors are important aspects of follow-up care. Methods: The Children's Oncology Group Long-Term Follow-Up Guidelines for Survivors of Childhood, Adolescent, and Young Adult Cancer (COG-LTFU Guidelines) were created by the COG to provide recommendations for follow-up care of survivors at risk for long-term complications. The male health task force of the COG-LTFU Guidelines, composed of pediatric oncologists, endocrinologists, nurse practitioners, an urologist, and a radiation oncologist, is responsible for updating the COG-LTFU Guidelines every 2 years based on literature review and expert consensus.

Results: This review summarizes current task force recommendations for the assessment and management of male reproductive complications after treatment for childhood, adolescent, and young adult cancers. Issues related to male health that are being investigated, but currently not included in the COG-LTFU Guidelines, are also discussed.

Conclusion: Ongoing investigation will inform future COG-LTFU Guideline recommendations for followup care to improve health and quality of life for male survivors.

\section{Female reproductive health after childhood, adolescent, and young adult cancers: guidelines for the assessment and management of female reproductive complications}

Metzger ML, Meacham LR, Patterson B, Casillas JS, Constine LS, Hijiya N, Kenney LB, Leonard M, Lockart BA, Likes W, Green DM

St. Jude Children's Research Hospital, Memphis, TN, USA

monika.metzger@stjude.org

J Clin Oncol 2013;31:1239-1247

Background: As more young female patients with cancer survive their primary disease, concerns about reproductive health related to primary therapy gain relevance. Cancer therapy can often affect reproductive organs, leading to impaired pubertal development, hormonal regulation, fertility, and sexual function, affecting quality of life.

Methods: The Children's Oncology Group Long-Term Follow-Up Guidelines for Survivors of Childhood, Adolescent, and Young Adult Cancer (COG-LTFU Guidelines) are evidence-based recommendations for screening and management of late effects of therapeutic exposures. The guidelines are updated every 2 years by a multidisciplinary panel based on current literature review and expert consensus.

Results: This review summarizes the current task force recommendations for the assessment and management of female reproductive complications after treatment for childhood, adolescent, and young adult cancers. Experimental pretreatment as well as posttreatment fertility preservation strategies, including 
barriers and ethical considerations, which are not included in the COG-LTFU Guidelines, are also discussed.

Conclusion: Ongoing research will continue to inform COG-LTFU Guideline recommendations for follow-up care of female survivors of childhood cancer to improve their health and quality of life.

These two outstanding papers summarize the Children's Oncology Group Long-Term Follow-Up Guidelines for Survivors of Childhood, Adolescent, and Young Adult Cancer for the assessment and management of male and female reproductive complications. The strength of these guidelines is that they are updated every 2 years by a multidisciplinary panel based on current literature review and expert consensus. The authors address all reproductive complications including hypogonadism, precocious puberty, reduced fertility, and sexual dysfunction as well as risk factors, clinical assessment, and interventions for each of the including treatment-associated complications. Additionally, controversial or investigational topics related to male and female health currently not incorporated in the Guidelines, are also presented.

\title{
New Cochrane review and more HPA axis after treatment of ALL
}

\section{Hypothalamic-pituitary-adrenal (HPA) axis suppression after treatment with glucocorticoid therapy for childhood acute lymphoblastic leukemia}

\author{
Gordijn MS, Gemke RJ, van Dalen EC, Rotteveel J, Kaspers GJ \\ Department of Pediatrics, Division of Oncology/Hematology, VU University Medical Center, Amsterdam, \\ The Netherlands \\ ms.gordijn@vumc.nl. \\ Cochrane Database Syst Rev 2012;5:CD008727
}

Background: Glucocorticoids play a major role in the treatment of acute lymphoblastic leukemia (ALL). However, supraphysiological doses may cause suppression of the hypothalamic-pituitary-adrenal (HPA) axis. HPA axis suppression resulting in reduced cortisol response may cause an impaired stress response and an inadequate host defense against infections, which remains a cause of morbidity and death. The exact occurrence and duration of HPA axis suppression after glucocorticoid therapy for childhood ALL are unclear.

Methods: To examine the occurrence and duration of HPA axis suppression after (each cycle of) glucocorticoid therapy for childhood ALL, the authors searched the Cochrane Central Register of Controlled Trials (in The Cochrane Library, issue 3, 2010), Medline/PubMed (from 1945 to July 2010) and EMBASE/Ovid (from 1980 to July 2010). In addition, they searched reference lists of relevant articles, conference proceedings and ongoing trial databases, and all study designs, except case reports and patient series with fewer than 10 patients, examining the effect of glucocorticoid therapy for childhood ALL on the HPA axis function. Two review authors independently performed the study selection. One review author performed the data extraction and 'risk of bias' assessment, which was checked by another review author.

Results: The authors identified seven studies (total number of participants $=189$ ), including one randomized controlled trial (RCT), which assessed the adrenal function. None of the studies assessed the HPA axis at the level of the hypothalamus, pituitary, or both. Due to substantial differences between studies, results could not be pooled. All studies had some methodological limitations. The included studies demonstrated that adrenal insufficiency occurs in nearly all patients in the first days after cessation of glucocorticoid treatment for childhood ALL. The majority of patients recovered within a few weeks, but a small number of patients had ongoing adrenal insufficiency lasting up to 34 weeks. In the RCT, the occurrence and duration of adrenal insufficiency did not differ between the prednisolone and dexamethasone arms. One study suggested that treatment with fluconazole prolonged the duration of adrenal insufficiency.

Conclusions: The authors conclude that adrenal insufficiency commonly occurs in the first days after cessation of glucocorticoid therapy for childhood ALL, but the exact duration is unclear. Since no data on 
the level of the hypothalamus and the pituitary were available they cannot draw any conclusions regarding those outcomes. Clinicians should consider prescribing glucocorticoid replacement therapy during periods of serious stress in the first weeks after cessation of glucocorticoid therapy for childhood ALL, to reduce the risk of life-threatening complications. However, more high-quality research is needed for evidence-based guidelines for glucocorticoid replacement therapy. Special attention should be paid to patients receiving fluconazole therapy, and perhaps similar antifungal drugs, as this may prolong the duration of adrenal insufficiency.

\section{Evaluation of adrenal reserve in children with acute lymphocytic leukemia treated with prednisone or dexamethasone}

Kuperman H, Odone Filho V, Cristofani LM, Assis de Almeida MT, Setian N, Damiani D

Pediatric Endocrine Unit, Instituto da Crianca, Pediatrics Department, São Paulo University Medical School,

São Paulo, Brazil

hkuperman@terra.com.br

Horm Res Paediatr 2012;78:73-80

Background: The aim of this study was to compare adrenal gland reserve in acute lymphocytic leukemia (ALL) patients 8 weeks after treatment with either prednisone (PRED) or dexamethasone (DEX) during the induction phase of therapy.

Methods: A double-blind comparative study of patients treated with PRED or DEX was performed. 16 patients received PRED $\left(40 \mathrm{mg} / \mathrm{m}^{2} /\right.$ day $)$ and 13 patients received DEX $\left(6 \mathrm{mg} / \mathrm{m}^{2} /\right.$ day $)$, both for 28 days. A low-dose adrenocorticotropic hormone test $\left(1.0 \mu \mathrm{g} / \mathrm{m}^{2}\right.$, i.v. $)$ was performed before and weekly for 8 weeks after abrupt cessation of glucocorticoid therapy. 16 children without ALL were used as controls to determine the cut-off peak cortisol level $(14.2 \mu \mathrm{g} / \mathrm{dl})$.

Results: Both groups (PRED and DEX) displayed similar mean peak cortisol levels before treatment and during the 8 weeks of evaluation $(\mathrm{p}=0.652)$. No relationship was observed between the incidence of infection/stress and peak cortisol level within each group, nor was there a difference in the frequency of infection/stress between groups $(\mathrm{p}=0.359)$. Although the patients presented variations in peak cortisol during the study period, no signs or symptoms of adrenal insufficiency were observed.

Conclusions: Patients who received PRED or DEX for 4 weeks showed similar adrenal reserves and infection rates for 8 weeks after abruptly stopping glucocorticoid therapy, suggesting that DEX, which is a better anti-leukemic drug than PRED, has similar adrenal suppression and recovery rates.

This excellent Cochrane review summarizes current evidence on HPA axis suppression after treatment of ALL. Supraphysiological doses of glucocorticoids play an important role within those therapy regimens, but there is still no evidence of how to handle the problem of HPA suppression after withdrawal of the oncologic steroid therapy. A high percentage of the patients have adrenal insufficiency on the basis of a low-dose ACTH stimulation test. However, most patients recover within a few weeks. The study by Kuperman et al. adds to these data by stating that there is no obvious difference between prednisone and dexamethasone in terms of adrenal insufficiency but also in terms of occurrence of infection. There is some limitation of this study due to relatively small patient numbers.

There is still no conclusive evidence on how best to monitor and treat patients after glucocorticoid treatment in ALL. Concepts range from no treatment, except stress dosing, to glucocorticoid substitution until the adrenal axis recovers. Our personal recommendation is: low dose glucocorticoid substitution until the adrenal axis recovers. In addition, there is still no final answer as to whether basal cortisol levels, the low-dose ACTH test, or other assessment methods are most valid to diagnose and monitor adrenal insufficiency. 


\section{Adrenal insufficiency and response to corticosteroids in hypotensive critically ill children with cancer}

Hebbar KB, Petrillo T, Fortenberry JD

Division of Critical Care, Department of Pediatrics, Emory University School of Medicine and Children's Healthcare of Atlanta at Egleston, Atlanta, GA, USA

kiran.hebbar@choa.org

J Crit Care 2012:27:480-487

Background: Assessment and treatment of adrenal axis function in critically ill pediatric patients with oncologic disorders have not been well elucidated. Critically ill children with oncologic disorders may be at increased risk for adrenal insufficiency (AI). The aim of this study was to evaluate the incidence of $\mathrm{AI}$ in a cohort of hypotensive patients with oncologic disorders and the correlation of AI with hemodynamic response to corticosteroids.

Methods: The authors performed a retrospective review of pediatric oncology patients with systemic inflammatory response syndrome and vasopressor-dependent shock receiving protocol-based adrenocorticotropin testing and steroid supplementation. Vasopressor dose requirements were evaluated before and after the initiation of corticosteroids. Absolute AI (AAI) was defined as baseline cortisol $<18$ $\mu \mathrm{g} / \mathrm{dl}$. Relative AI (RAI) was defined as $\Delta$-cortisol $<9 \mu \mathrm{g} / \mathrm{dl}$.

Results: 20 patients received adrenocorticotropin testing; primary diagnoses were 13 hematologic malignancies and 7 solid tumors. The median age was 132 months (range 4-224). The median fluid resuscitation was $80 \mathrm{ml} / \mathrm{kg}$ (range $60-120)$, with $10(50 \%)$ of 20 patients requiring mechanical ventilation for a median of 12 days. Overall, $10(50 \%)$ of 20 patients met the definition for RAI, $15(75 \%)$ of 20 met the definition for AAI, and $18(90 \%)$ of 20 had either AAI or RAI. All patients received steroids: 18/20 $(90 \%)$ were on dopamine and $17 / 20(85 \%)$ were on norepinephrine. After the introduction of steroids, doses of norepinephrine and dopamine were significantly reduced by $4 \mathrm{~h}(\mathrm{p}=0.0001)$.

Conclusions: Both RAI and AAI were common in this cohort of critically ill pediatric patients with oncologic disorders. Introduction of steroids was associated with a significant reduction in vasopressors dose.

Critical illness-related corticosteroid insufficiency (CIRCI) is highly prevalent [7, 8]. However, methods and cut-off values to determine $\mathrm{CIRCI}$ in children are still a subject of debate. In addition, results on the outcomes of patients with $\mathrm{CIRCI}$ who are treated with glucocorticoids are inconclusive.

This retrospective study by Hebbar et al. on $\mathrm{CIRCI}$ found a high incidence of absolute (AAI) and relative adrenal insufficiency (RAI) in a cohort of hypotensive critically ill children with malignancies. The definitions for AAl and RAl were based on suggested levels from the International Surviving Sepsis Campaign Guidelines Committee guidelines. AAI in the case of catecholamine-resistant septic shock is assumed at a random total cortisol concentration $<18 \mu \mathrm{g} / \mathrm{dl}(496 \mathrm{nmol} / \mathrm{l})$, whereas a post 30 - or 60 -min ACTH stimulation test increase in cortisol of $<9 \mu \mathrm{g} / \mathrm{dl}(248 \mathrm{mmol} / \mathrm{l})$ has been used to define RAI [9]. The incidence of AAI did not correlate with prior corticosteroid use as chemotherapy. Exogenous corticosteroid supplementation was associated with a significant reduction in vasopressor duration and dose.

\section{Important for clinical practice}

\section{Growth in children receiving contemporary disease-specific therapy for Crohn's disease}

Malik S, Mason A, Bakhshi A, Young D, Bishop J, Garrick V, McGrogan P, Russell RK, Ahmed SF

Department of Child Health, University of Glasgow, Bone and Endocrine Research Group, Royal Hospital for Sick

Children, Glasgow, UK

Arch Dis Child 2012;97:698-703

Background: Growth retardation may occur in children with Crohn's disease (CD). Current therapy for $\mathrm{CD}$ in the UK is less likely than previously to involve the use of long-term glucocorticoids. It is unclear 
whether recent therapeutic advances have improved the growth of children with CD. The authors aimed to assess the frequency of short stature and poor growth and their relationship to disease course and therapy in children with CD. Despite advances in therapy, short stature and slow growth continue to be encountered in children with CD. There is a need for simple and consistent definitions of growth that can identify poor growth in children with chronic disease.

Methods: The anthropometric and treatment details of 116 children (68 male) with CD with a mean (range) age at diagnosis of 10.8 years (4.9-15.5) and a mean age at maximum follow-up (MF) of 15.4 years (9.4-19.3) were studied retrospectively at diagnosis (T0), at 1 (T1), 2 (T2) and 3 years (T3) after diagnosis and at MF.

Results: At T0, mean height SD score (HtSDS) was -0.5 ( -3.3 to 2.6) compared to a mid-parental HtSDS of $0.2(-2.0$ to 1.4$)(\mathrm{p}=0.002)$. At T1, T2, T3 and MF, mean HtSDS was -0.6 ( -4.8 to 7.8$),-0.6(-2.9$ to 2.2$),-0.7$ (-3.6 to 2.5 ) and -0.5 ( -3.5 to 2.9 ), respectively. Mean Ht velocity (HV) SDS at T1, T2, T3 and MF was -1.4 (-7.4 to 7.4$),-0.6$ ( -7.5 to 6.1$),-0.1$ (-6.6 to 7.6$)$ and 0.6 ( -4.8 to 7.8$)$, respectively $(\mathrm{p}<0.05)$. In final models, HtSDS was associated negatively with the use of prednisolone $(\mathrm{p}=$ $0.0001)$, azathioprine $(\mathrm{p}=0.0001)$, methotrexate $(\mathrm{p}=0.0001)$ and weight SDS (WtSDS) $(\mathrm{p}=0.0001)$. HVSDS was associated positively with age $(\mathrm{p}=0.0001)$ and WtSDS $(\mathrm{p}=0.01) . \Delta \mathrm{HtSDS}$ was associated negatively with use of prednisolone $(\mathrm{p}<0.02)$.

Conclusion: Although current therapy for CD is associated with improved rate of growth for the first few years, a substantial proportion of children remain short. This study also highlights the need for consistency in describing growth in children with chronic diseases.

Children with Crohn's disease have growth failure and delayed puberty due to the disease per se and/ or as a side effect of glucocorticoid therapy. During the last decade, glucocorticoid treatment has been partly replaced by other immunomodulatory drugs. However, a substantial proportion of children continue to show poor growth irrespective of therapeutic regimen. The authors suggest that these patients may benefit from specific growth-promoting therapy or novel therapeutic strategies.

\section{New hope}

\section{Normal height and weight in a series of ambulant Duchenne muscular dystrophy patients using the 10-day on/10-day off prednisone regimen}

Ten Dam K, de Groot IJ, Noordam C, van Alfen N, Hendriks JC, Sie LT

Department of Pediatric Neurology, Donders Institute for Brain, Cognition and Behaviour, Radboud University Nijmegen-Medical Centre, The Netherlands

Neuromuscul Disord 2012;22:500-504

Background: Prednisone treatment delays the progressive course of Duchenne muscular dystrophy (DMD). The aim of this study was to determine the influence of the 10-day on/10-day off treatment on height and weight.

Methods: The authors retrospectively reviewed the growth and weight charts of DMD patients born between 1988 and 2006 (patients between 4 and 9 years of age, being able to walk in the home situation). 47 patients were eligible for further analysis and divided into two groups: 33 patients treated with prednisone and 14 non-prednisone-treated patients.

Results: Results of a median follow-up of 57 months (range 27-146) are described. By using linear mixed models this study demonstrates that height and body mass index in prednisone-treated DMD patients with a 10/10 regimen are not significantly different compared to untreated DMD patients.

Conclusions: We cautiously conclude that the alternating prednisone regimen has no apparent side effects on weight and height in the ambulatory phase of DMD.

Glucocorticoid therapy has been shown to have beneficial effects on the progression of Duchenne muscular dystrophy (DMD). The results of this study contrast with previous reports that an alternating regimen does not reduce side effects on weight and BMI. However, several other studies have confirmed that a daily dose of glucocorticoids affects growth and weight. It is of note that children 
with DMD are also at risk for overweight and growth failure without glucocorticoid treatment. Probably, the number of patients, the used statistical methods and the time of follow-up might account for the different results among the studies investigating 10-day on/10-day off regimens. Additional data are needed to confirm the data reported by the authors.

\section{Confirmation of previous results}

\section{Prevalence of overweight in Dutch children with Down syndrome}

Van Gameren-Oosterom HB, van Dommelen P, Schonbeck Y, Oudesluys-Murphy AM, van Wouwe JP, Buitendijk SE Departments of Child Health, Netherlands Organization for Applied Scientific Research, TNO, Leiden, Netherlands helma.vangameren@tno.nl Pediatrics 2012;130:e1520-e1526

Background: Prevalence of overweight in children is increasing, causing various health problems. This study aims to establish growth references for weight and to assess the prevalence rates of overweight and obesity in a nationwide sample of Dutch children with Down syndrome (DS), taking into account the influence of comorbidity.

Methods: In 2009, longitudinal growth data from Dutch children with trisomy-21 who were born after 1982 were retrospectively collected from medical records of 25 Dutch regional specialized DS centers. 'Healthy' was defined as not having concomitant disorders or having only a mild congenital heart defect. Weight and BMI references were calculated by using the LMS method and prevalence rates of overweight and obesity by using cut-off values for BMI as defined by the International Obesity Task Force. Differences in prevalence rates were tested by multilevel logistic regression analyses to adjust for gender and age.

Results: Growth data of 1,596 children with DS were analyzed. Compared with the general Dutch population, healthy children with DS were more often overweight $(25.5$ vs. $13.3 \%$ in boys, and 32.0 vs. $14.9 \%$ in girls) and obese (4.2 vs. $1.8 \%$, and $5.1 \%$ vs. $2.2 \%$, respectively). Prevalences of overweight did not differ significantly between DS children with or without concomitant disorders.

Conclusions: Dutch children with DS have alarmingly high prevalences of overweight and obesity during childhood and adolescence. Healthcare professionals should be aware of the risk of overweight and obesity in children with DS to prevent complications.

Overweight and obesity are increasing problems around the world, particularly in the developed countries of the Western hemisphere. Styles et al. [10] in 2002 found that obesity amongst adolescents with DS had increased compared with the data reported by Cronk et al. [11] in 1988. Styles et al. reported about $30 \%$ of adolescents with DS as being overweight. This is similar to the current findings of van Gameren-Oosterom and colleagues, who addressed the high percentage of overweight children of the age group of 2-6 years and the impact of hypothyroidism and congenital heart defects on weight development. The study also emphasizes the importance of early overweight in children with DS with a view to the occurrence of obstructive sleep apnea (OSA), musculoskeletal disorders, cardiovascular consequences, and impaired general physical condition later in life. The authors seek to increase awareness of the risks of early overweight among both healthcare professionals and parents and patients to enable early detection of inappropriate weight gain, initiate appropriate interventions, and prevent the long-term sequelae. The centiles used in the study are available online at www.tno.nl/growth. Overweight and obesity cause both psychological and physical health problems in children, such as low self-esteem, depressive symptoms, lower general physical condition, and metabolic complications. It is not known whether all these consequences of overweight and obesity in children in the general population are to be expected in children with DS because no data have yet been published on the effect of overweight and obesity in children with DS. Parents need to know what a healthy weight is for their child with DS. With this in mind, they can support their child to achieve and maintain a healthy weight. These approaches to prevent excessive weight gain are an important task for professionals involved in the care for children with DS. 
1. Donadieu J, Rolon MA, Pion I, Thomas C, Doz F, Barkaoui M, et al: Incidence of growth hormone deficiency in pediatric-onset Langerhans' cell histiocytosis: efficacy and safety of growth hormone treatment. J Clin Endocrinol Metab 2004;89:604-609.

2. Kaltsas GA, Powles TB, Evanson J, Plowman PN, Drinkwater JE, Jenkins PJ, et al: Hypothalamo-pituitary abnormalities in adult patients with Langerhans' cell histiocytosis: clinical, endocrinological, and radiological features and response to treatment. J Clin Endocrinol Metab 2000;85:1370-1376.

3. Maghnie M, Bossi G, Klersy C, Cosi G, Genovese E, Arico M: Dynamic endocrine testing and magnetic resonance imaging in the long-term follow-up of childhood Langerhans' cell histiocytosis. J Clin Endocrinol Metab 1998;83:3089-3094.

4. American Academy of Pediatrics Section on Hematology/Oncology Children's Oncology Group: Long-term follow-up care for pediatric cancer survivors. Pediatrics 2009;123:906-915.

5. Brougham MF, Crofton PM, Johnson EJ, Evans N, Anderson RA, Wallace WH: Anti-mullerian hormone is a marker of gonadotoxicity in pre- and postpubertal girls treated for cancer: a prospective study. J Clin Endocrinol Metab 2012;97:2059-2067.

6. Gracia CR, Sammel MD, Freeman E, Prewitt M, Carlson C, Ray A, et al: Impact of cancer therapies on ovarian reserve. Fertil Steril 2012;97:134-140.e1.

7. Marik PE: Critical illness-related corticosteroid insufficiency. Chest 2009;135:181-193.

8. Indyk JA, Candido-Vitto C, Wolf IM, Venkataraman S, Munoz R, Saladino RA, et al: Reduced glucocorticoid receptor protein expression in children with critical illness. Horm Res Paediatr 2013;79:169-178.

9. Dellinger RP, Levy MM, Carlet JM, Bion J, Parker MM, Jaeschke R, et al: Surviving Sepsis Campaign: international guidelines for management of severe sepsis and septic shock: 2008. Crit Care Med 2008;36:296-327.

10. Styles ME, Cole TJ, Dennis J, Preece MA: New cross-sectional stature, weight, and head circumference references for Down's syndrome in the UK and Republic of Ireland. Arch Dis Child 2002;87:104-108.

11. Cronk C, Crocker AC, Pueschel SM, Shea AM, Zackai E, Pickens G, et al: Growth charts for children with Down syndrome: 1 month to 18 years of age. Pediatrics 1988;81:102-110. 
